\section{PULMONARY PHTHISIS ;}

SOME REMARKS AS TO ITS PATIOLOGY AND ITS CURABILITY IN EARLY STAGES, PARTICLLARLY BY THE USE OH HOT AIR INHALATIONS.

By T. CRANSTOUN CHARLES, M.D.

AT the meeting of the British Medical Association in Leeds two years ago I read a paper on the hot air treatment of pulmonary phthisis, and as it so happened that all the cases $I$ had had under treatment were in an early stage of the disease, I had only a series of very successful results to communicate. Since then, however, my experience of the method has been greatly extended, and I have come to the conclusion that only in very early cases can any benefit be expected from it. A great amount of interest is now being directed towards the cure of this disease, particularly owing to Dr. Koch's valuable endeavours in this direction; and should his efforts be crowned with success, not only in early but also in somewhat advanced cases, then a great and good work will have been accomplished, and Dr. Koch will truly deserve to be ranked as one of the greatest benefactors of the human race.

The apparently esseutial characteristic in the pathology of phthisis consists, it is believed, in a caseous necrosis, the typical phenomena of the disease being produced as the result of the influence of a special morbid poison. Its starting point seems to be the lodgment of a few tubercle bacilli in the finer bronchial tubes or in the alveoli of the lungs, leading to the development of the miliary tubercles, and a consequent inflammation of the bronchioles extending to the proper parenchyma of the lungs. The caseous necrosis, therefore, depends on the growth and development of these special tubercle bacilli ; indeed, every form of tuberculosis of the lung may be said to have received its first impulse from the tubercle bacilli. In their growth these bacilli evolve certain chemical products which are highly injurious to the tissues with which they come in contact-products also which are not of necessity confined in their action to the surfaces and canals where they are generated, but may extend to other parts of the organism, and excite there inflammatory changes of different kinds. The lesion, however, is not usually a penetrating one, at least in the early stages, but rather tends to spread superficially along the air passages and lymphatics-a progressive infection accordingly depending upon the multiplication of the bacilli. It is further known that the character of the disease depends in part on the quantity and quality or degree of virulence of these bacilli, and partly on the susceptibility of the lungs of some individuals to their lodgment and development, while a progressive infection is due to their multiplication. Where, for example, the normal tissue resistance is too great, these organisms can obtain no resting place, or, if they do, their development is of short duration. In some individuals, however, the tissue resistance is less powerful, and accordingly a morbid tubercular process is set up, leading to the condition known as fibcoid phthisis. In this variety the tissue resistance is greater than in the next, and accordingly its course is not so rapid; the patients also are generally males, and their age more advanced. Where, again, there is still less of this tissue resistance, the ordinary caseous phthisis is established, an exceptional form occasionally occurring-the so-called "acute general tuberculosis,"-where the susceptibility is very great, or, in other words, the tissue resistance very feeble, and in which the bacilli develop freely, make their way in great numbers into the blood, and by its means effect a lodgment in many organs simultaneously, leading to the outbreak of innumerable tubercles. Upon the degree of virulence of these bacilli, owing chiefly as it does to their quantity and quality, depends the type of tubercle produced; for Baumgarten has shown that while inoculation of animals with pure cultivations of tubercle bacilli induces the development of the lymphatic tubercles, inoculation with bacilli whose virulence has been weakened by the action of heat will only lead to the development of the epithelioid tubercles-that is, an abundant impregnation with the active bacilli tends to the building up and rapid degeneration of lymphatic tubercles; whilst an impregnation with a small amount of bacilli whose vitality has been impaired will lead to the formation of epitheliod tubercles, with the accompanying formation of much connective tissue, and its subsequent shrinking as the result of the irritation.

As Dr. Coats puts it, in his admirable lectures on the Pathology of Phthisis, to which I am much indelted, pulmonary consumption is a disease of surfaces, channels, and tubes. Beginning in the finer bronchial tubes, the disease may extend by the air passages or by the lym. phatics, probably by both, but chiefly, probably by the airpassages in the caseous form, and by the lymphaties in the tibroid. A certain susceptibility, we have said, must be present to admit of one or other of these diseased processes, and this susceptibility, there is every reason to believe, may be hereditary or it may be developed. The pre-existence of certain affections of the lungs, for example, we know undoubtedly plays an important part in bringing about a greater liability to the invasion of these bacilli, particularly when the vitality of the tissues is already lowered by the respiration of vitiated air.

Different methods have been employed to destroy these tubercle bacilli, and it is a very fortunate thing that they have comparatively little vitality and offer little resistance to the action of reagents. They are easily destroyed, for instance, by such bodies as carbolic acid, corrosive sublimate, salicylic acid, acetic acid, \&c., and accordingly various attempts have been made to kill them in the lungs. All these attempts, nevertheless, it must be confessed, have proved more or less futile, so far, at least, as the cure of the patient was concerned. With the same object in view Dr. Louis Weigert of Berlin some years ago introduced the inhalation of hot air. Koch and other observers had shown that tubercle bacilli are specially sensitive to the influence of temperature. They develop best about $37.5^{\circ} \mathrm{C}$, while their growth is weakened at $38^{\circ} 5^{\circ}$, and ceases at $42^{\circ}$, and at any higher temperature than this their vitality is quite destroyed. Dr. L. Weigert finding that it was possible to sterilise these bacilli by means of the discontinuous periodic action of heated air, and that a man could breathe very hot air for prolonged periods without suffering any injury, then convinced himself of the possibility of effecting a sterilisation of the bacilli in the lung substance itself. This is done by the daily inhalation of heated dry air at a temperature of $200^{\circ}$ to $250^{\circ} \mathrm{C}$. for about two hours at a time, and for a lengthened period extending over several months.

Without going into the question of the temperature of this heated air when it reaches the lungs, I shall confine myself to saying that in early cases of pulmonary phthisis its use certainly caused the number of bacilli in the sputum to diminish greatly, and even in a few cases to disappear entirely. But undoubtedly another great advantage noted was the forced exercise of the lungs induced by it. This lung-gymnastics proves of essential service not only to the lungs, but to the well-being of the body generally, for collapse of the pulmonary lobules, with its attendant evil consequences, is thereby prevented, while, at the same time, the inspiratory muscles undergo a marked development. I therefore attempted to sterilise or destroy the tubercle bacilli by the inhalation of hot air, and at the same time made an effort to check any further invasion of the tissues by the bacilli by inuproving the general tone of the tissues. It is to be carefully borne in mind that a susceptibility exists, and that every means consequently must be adopted to lessen or remove this as far as possible, as, for example, a good and varied diet, moderate exercise in the open air, and change of air where possible-the more elevated and drier the situation the better,-plenty of sunlight also, massage, cold douches, and the like. In connexion with the function of digestion in my phthisical patients, I should mention that whenever I found it impaired at all, as the result of a catarrhal condition of the stomach - which is so frequently the case, - I made a point of beginning the treatment by washing out that viscus, employing in succession for that purpose a warm saline, a warm alkaline, and, lastly, a warm acid solution. To this I attach some importance, as it is essential to success that the stomach be in a condition to perform its duty in digestion. The decided and often inmediate improvement I obtained in this way was sometimes remarkable. Some patients require this washing process repeated several times, but others do not. And not only is it of importance that the stomach should be able to digest the food, but its activity also serves as a safeguard to the intestines; for it is the passage of the tubercle bacilli through the stomach that precedes the intestinal ulcerations; but as long as the gastric juice is possessed of 
its normal acidity, the bacilli in passing through have their vitality destroyed or greatly inhibited. Where, however, there is much gastric catarrh, the mucous membrane being thickly covered with mucus, and the hydrochloric acid greatly diminished in amount, as I have frequently observed, then the bacilli in the swallowed sputum may pass safely through and reach the intestine, there to effect lodgment in the gut, and bring about tubercular ulceration-a condition of things of much too frequent occurrence, seeing that it is present in from one-half to two-thirds of the fatal cases of pulmonary tubercle.

As I have already stated, the only success I have obtained by the use of the hot-air treatment has been in early cases of the disease. It is therefore of importance to begin it as early as possible. By doing so we may probably prevent any marked invasion of the blood by the bacilli, with the acute trberculosis consequent thereupon, as also the possibility of the stomach and intestines being affected with the disease, as referred to above. I shall enter into no description of the apparatus employed for heating the air, but shall linit myself to stating that it has been used by healthy men for prolonged periods without producing any bad results, while in early cases of pulmonary phthisis I have noted the following after its prolonged use : An increase in the vital capacity of the lungs, with a considerably increased expansion of the thorax, varying from an inch to three inches; a gradual disappearance of the atrophic condition of the external thoracic inspiratory muscles, the diaphragm also becoming more vigorous in its action, the inspirations accordingly being rendered deeper and more powerful; a diminution in the bronchial catarrh, the expectoration, though increased at first, being afterwards diminished, and the cough finally disappearing; and a general improvement in the appetite and health, and an increase in body weight. The bacilli, although considerably increased at first, undergo a marked diminution with the continnance of the inhalations-indeed, they may finally disappear entirely. This examination for bacilli in the sputum should never be overlooked, as their presence is one of the most, if not the most, important sign of the disease, and not one, but a series of examinations of the same sputum should be made, of which a considerable quantity should be collected and mixed thoroughly. I generally direct the patient to make a practice of spitting into a small wide-mouthed bottle containing a few drops of chloroform or a piece of menthol. Indeed, I think the sputum of phthisical people generally should not be distributed broadcast, as is the usual habit, but ought to be collected in some such way as I have suggested, and afterwards destroyed by fire or antiseptics.

The method I pursue in the examination is generally the following. A little of the mixed sputum is spread out between two thin cover glasses, which are then separated carefully and passed several times through the flame of a spirit lamp, so as to coagulate the albumen and fix the sputum to the glass. The staining fluid I prefer is Neelsen's: magenta, 1 ; carbolic acid (5 per cent.), 100 ; absolute alcohol, 10 . A little of this fluid is heated in a small porcelain dish till steam rises, when the cover glasses with their adherent sputum are immersed in it, the dish covered and laid aside for three minutes. The cover glasses are now lifted out and placed at once in a 25 per cent. solution of sulphuric acid, in which they are to be moved about freely for a short time so as to decolourise all but the tubercle bacilli, when they are to be placed finally for a couple of minutes in a dark-blue solution of methylene blue so as to obtain a contrast stain, and lastly washed in distilled water, dried, and mounted in thick balsam. It quickens the process somewhat to dissolve the methylene blue in the sulphuric acid. In cases of fibroid phthisis there is often some difficulty in discovering the bacilli. Here, as well as in cases where bacilli are suspected to be present but cannot readily be found, several examinations require to be made; but I find that by spreading out a considerable quantity of the sputum between two clean sheets of plate glass little translucent masses may often be noticed, which when carefully removed and examined will usually show the presence of bacilli.

The experience I have had with this method of treatment disposes me to recommend its use only in the early stages of pulmonary phthisis-cases where the pulmonary tuberculisation is not far advanced, and the lung substance only slightly involved, and not at all broken down; where, in short, the bacilli are most recently deposited, and accord ingly most superficial. Indeed, where there is a strons suspicion of tubercle setting in, although no positive physical signs of tubercular deposit may be capable of detection; where, for example, there is hemoptysis which we cannot refer to any other source-as from the nose, mouth, gums, throat, larynx, trachea, stomach, or from structural alterations in the terminal pulmonary bloodvessels of elderly people with an arthritic diathesis (Clark); or during severe attacks of acute bronchial catarrh with violent coughing, or after severe bodily exertion or the inhalation of highly irritant gases, - then, all these probable causes being cautiously eliminated, it would be advisable to adopt its use.

That a bronchial catarrh, particularly if confined to the apex of the lung, or spreading there and inducing a catarrhal pneumonia - a pneumonia localised in the upper lobe, or a catarrhal pneumonia in the lower lobe-may often be followed by tubercle, we have unfortunately too many examples. Now it so happens that this hot-air method provides us with an admirable means of treating many obstinate forms of bronchitis; and I have frequently used it in such cases with the most marked benefit. No doubt many of the milder cases of pulmonary phthisis may eventually recover of themselves, particularly if the conditions are at all favourable; but the course is necessarily very slow, and liable to many accidents.

(To be concluded.)

\section{R H I N O P L A S T Y.}

BY SURGEON-MAJOR D. F. KEEGAN, M.D. DUB., RESIDENCY SURGEON, INDORE, CENTRAL INDIA.

THE conditions under which the surgeon in India performs rhinoplasty differ somewhat from those under which he does the same operation in Europe. In Europe, in the majority of cases, rhinoplasty is performed in order to repair the ravages and disfigurement caused by lupus or tertiary syphilis, and it is but seldom that plastic surgery is called upon to make good the damage done to a nose by accident or mutilation. In India, on the cther hand, in the vast majority of cases, rhinoplasty is performed to hide the ghastly disfigurement caused by mutilation, and our patients are almost invariably young, healthy, and robust. During my service in India I think I must have performed between forty and fifty rhinoplastic operations, and in every single case they were undertaken to repair the damage inflicted by mutilation. It will be conceded by most surgeons who have had considerable experience in rhinoplasty that the Indian method of operation possesses many advantages over both the Taliacotian and the French plans of procedure. The French method I need hardly say consists in fasbioning a new nose by means of flaps taken from the cheeks. The Indian operation, as described and figured in English surgical text-books, is, however, susceptible of much improvement, and it is with the object of bringing this improvement to the notice of the profession in India and in this country through the medium of THE LANCET that $I$ write this short paper. The operation which I have performed of late years as the outcome of considerable experience of rhinoplasty is, however, only applicable in its fullest extent to those cases of loss of nose in which the skin and tissues which clothe the nasal bones have been left undamaged. All surgeons who have had much experience in making new noses are, I think, agreed, that the principal difficulties and drawbacks of the operation centre in the formation of the columna, and in obviating the continued tendency to contraction in the anterior nares of the newly fashioned nose, a tendency which continues for several months after operation. The flap which I raise from the forehead differs considerably in outline from that which we see generally figured in English surgical text-books, and although German surgeons have to some extent adopted the same method of obviating the contraction of the anterior nares as we have pursued for some years at Indore, still I feel I need not offer any apology for bringing the subject to the notice of my English readers. This method of forming new noses has been worked oxt independently by my assistant, Mr. Gunput Singh, and mygelf, but we disclaim for it any title to originality, for he would be bold indeed who would assert that he had devised a perfectly new operation in the changeful field of plastie 